\title{
Rationale of Radioembolization in Intermediate and Advanced Stage Hepatocellular Cancer
}

\author{
Cigdem Soydal \\ Department of Nuclear Medicine, Ankara University Medical School, Ankara, Turkey
}

Received: August 09, 2014; Accepted: August 20, 2014; Published: September 01, 2014

*Corresponding author: Cigdem Soydal, Department of Nuclear Medicine, Ankara University Medical School, Ankara, Turkey, E-mail: csoydal@yahoo. com

Rational of treatment for Barcelona Cancer Liver Clinic (BCLC) intermediate and advanced staged hepatocellular carcinoma (HCC) patients are still unclear [1].

Transarterial Chemoembolization (TACE) has been recommended to choice of treatment for intermediate stage patients in several guidelines [2,3]. Median overall survival in this patient group might increase to 20 months from 16 with TACE [2]. However because intermediate stage has a large range of patients with different tumor burden and liver functions, outcome of the treatment may vary. In advanced stage patients, sorafenib is the recommended treatment with different results due to individual sensitivity and severity of adverse effects. Thus, intermediate and advanced stage HCCs has been the subject of experimenting new treatment strategies. Radioembolization (RE) with Y-90 resin or glass microspheres has been performed in intermediate and advanced stage HCC patients in many center as well as ours. Worldwide, over 15000 RE have been performed. Two types of microspheres are available in market. Resin microspheres (Sirtex, Syndey, New South Wales, Australia) have a diameter of $32 \pm 10 \mu \mathrm{m}$ and are approved by FDA in USA in the treatment of unresectable HCC and also have CE marking in European Countries. Glass microspheres (TheraSphere, Kanata, Ontario, Canada) have a diameter of $25 \pm 10 \mu \mathrm{m}$ and have CE marking in European Countries.

Although randomized clinical trials have not been completed yet, current literature on RE has promising results with good tolerability and high response rates [4,5]. Safety of RE is strongly correlated with successof pretreatmentangiographyandocclusion of branches of hepatic artery that supply to extrahepatic organs. During this angiography procedure Macro Aggregated Albumin (MAA) labeled with Tc-99m injected into the hepatic artery and subsequently planar and SPECT scintigraphic images of abdomen and thorax was obtained. In these images shunt fraction of liver to lung is calculated to evaluation of appropriateness of patients for treatment. However it has been reported in the literature, and it is also our experience, intrahepatic distribution of Tc-99m MAA uptake and Y-90 microspheres are not corresponded [6,7].

A prospective study which focused on safety and efficacy of RE in intermediate or advanced stage HCC patients has reported
13 months time to progression after RE [8]. However liver decompensation rates have been found significant with 35\% in 6 months. Because most HCC patients have a chronic liver disease calculation of given activity is highly important to avoid liver decompensation after RE. Different dose calculation methods have been investigating such as partition, the empirical and body surface are model [9]. Rationale of the RE to achieve tumoricidal doses to the tumor which is highly correlated with vascularity of tumor without to aggravate underlying liver disease.

RE has several advantages in selected intermediate and advanced stage HCC patients. However its routine usage seems to have some time to settle of gold standard dose calculation method to prevent aggravation of underlying liver disease. Additionally possibility of hypovascular tumors should keep in mind during evaluation of patients.

\section{References}

1. Bolondi L, Burroughs A, Dufour JF, Galle PR, Mazzaferro V, Piscaglia $\mathrm{F}$, et al. Heterogeneity of patients with intermediate (BCLC B) Hepatocellular Carcinoma: proposal for a subclassification to facilitate treatment decisions. Semin Liver Dis. 2012; 32(4):348-59. doi: 10.1055/s-0032-1329906

2. European Association For The Study of The Liver1; European Organisation For Research And Treatment Of Cancer. EASL-EORTC clinical practice guidelines: management of hepatocellular carcinoma. J Hepatol. 2012; 56(4):908-43. doi: 10.1016/j.jhep.2011.12.001.

3. Bruix J, Sherman M; American Association for the Study of Liver Diseases. Management of hepatocellular carcinoma: an update. Hepatology. 2011;53(3):1020-2. doi: 10.1002/hep.24199.

4. Kennedy AS, Salem R. Radioembolization (yttrium-90 microspheres) for primary and metastatic hepatic malignancies. Cancer J. 2010;16(2):163-75. doi: 10.1097/PP0.0b013e3181d7e8cf.

5. Nicolay NH, Berry DP, Sharma RA. Liver metastases from colorectal cancer: radioembolization with systemic therapy. Nat Rev Clin Oncol. 2009; 6(12): 687-97. doi: 10.1038/nrclinonc.2009.165.

6. Gupta T, Virmani S, Neidt TM, Szolc-Kowalska B, Sato KT, Ryu RK, et al. MR tracking of iron-labeled glass radioembolization microspheres during transcatheter delivery to rabbit VX2 liver tumors: feasibility study. Radiology. 2008; 249(3): 845-54. doi: 10.1148/ radiol. 2491072027 . 
7. Kucuk ON, Soydal C, Araz M, Ozkan E, Aras G. Evaluation of the response to selective internal radiation therapy in patients with hepatocellular cancer according to pretreatment (99m)Tc-MAA uptake. Clin Nucl Med. 2013; 38(4): 252-5. doi: 10.1097/RLU.0b013e31828162e8.

8. Mazzaferro V, Sposito C, Bhoori S, Romito R, Chiesa C, Morosi C, et al. Yttrium-90 radioembolization for intermediate-advanced hepatocellular carcinoma: a phase 2 study. Hepatology. 2013; 57(5): 1826-37.
9. Garin E, Rolland Y, Boucher E, Ardisson V, Laffont S, Boudjema K, et al. First experience of hepatic radioembolization using microspheres labelled with yttrium-90 (TheraSphere): practical aspects concerning its implementation. Eur J Nucl Med Mol Imaging. 2010; 37(3): 453-61. doi: 10.1007/s00259-009-1279-6. 